\title{
Lipopolysaccharide of Yersinia pestis, the Cause of Plague: Structure, Genetics, Biological Properties
}

\author{
Y. A. Knirel ${ }^{1 *}$, A. P. Anisimov ${ }^{2}$ \\ ${ }^{1}$ Zelinsky Institute of Organic Chemistry, Russian Academy of Sciences, Leninsky prospect, 47, \\ Moscow, Russia, 119991 \\ 2 State Research Center for Applied Microbiology and Biotechnology, Obolensk, Moscow Region, \\ Russia, 142279 \\ *E-mail: knirel@ioc.ac.ru \\ Received 01.06.2012 \\ Copyright $\odot 2012$ Park-media, Ltd. This is an open access article distributed under the Creative Commons Attribution License, which permits \\ unrestricted use, distribution, and reproduction in any medium, provided the original work is properly cited.
}

\begin{abstract}
The present review summarizes data pertaining to the composition and structure of the carbohydrate moiety (core oligosaccharide) and lipid component (lipid A) of the various forms of lipopolysaccharide (LPS), one of the major pathogenicity factors of Yersinia pestis, the cause of plague. The review addresses the functions and the biological significance of genes for the biosynthesis of LPS, as well as the biological properties of LPS in strains from various intraspecies groups of $Y$. pestis and their mutants, including the contribution of LPS to the resistance of bacteria to factors of the innate immunity of both insect-vectors and mammal-hosts. Special attention is paid to temperature-dependent variations in the LPS structure, their genetic control and roles in the pathogenesis of plague. The evolutionary aspect is considered based on a comparison of the structure and genetics of the LPS of $Y$. pestis and other enteric bacteria, including other Yersinia species. The prospects of development of live plague vaccines created on the basis of $Y$. pestis strains with the genetically modified LPS are discussed.

KEYWORDS lipopolysaccharide; lipid A; plague; Yersinia pestis; immune response; antibiotic resistance

ABBREVIATIONS CAMP - cationic antimicrobial peptides; LPS - lipopolysaccharide; NHS - normal human serum; Ara4N - 4-amino-4-deoxy-L-arabinose; Gal - galactose; Glc - glucose; GlcN, GlcNAc - glucosamine, $N$-acetylglucosamine; DD-Hep, LD-Hep - D-glycero-, L-glycero-D-manno-heptose; Kdo - 3-deoxy-D-mannooct-2-ulosonic acid; Ko-D-glycero-D-talo-oct-2-ulosonic acid; PEtN - phosphoethanolamine; UndP, UndPP undecaprenyl phosphate, diphosphate.
\end{abstract}

\section{INTRODUCTION}

In the past decade, significant progress has been achieved in the study of the chemical structure, biosynthesis, and biological role of the lipopolysaccharide (LPS) as one of the pathogenicity factors of the bacteria Yersinia pestis, the cause of plague. Significant progress in this area has been achieved in the past decade after the September 2001 terrorist attacks, horrific events that prompted thorough research in dangerous pathogens that could potentially find application in the realm of biological terrorism.

The Yersinia genus belongs to the Enterobacteriaceae family. Unlike other representatives of this family, including two enteropathogenic Yersinia species, Y. pseudotuberculosis and Y. enterocolitica, which cause chronic intestinal infections, $Y$. pestis cannot exist under ambient conditions for a long time. The plague microbe circulates in natural foci, including populations of over 200 species of rodent hosts (ground squirrels, marmots, gerbils, voles, pikas, etc.) and insect vectors (over 80 flea species) [1-5]. A high mortality rate due to plague in rodents is a prerequisite for the continuous transmission of $Y$. pestis in nature.

The $Y$. pestis species contains both genotypically and phenotypically different variants [5, 6]. Strains of the main subspecies, Y. pestis subsp. pestis, belonging to biovars antiqua, medievalis, orientalis, and intermedium are virulent for humans and guinea pigs. Three plague pandemics are believed to have been caused by strains of each of the first three biovars. It has been proposed that strains of biovars altaica, caucasica, hissarica, ulegeica, talassica, xilingolensis, ginghaiensis, and angola, which are highly virulent in rodent hosts (various vole species belonging to the genus Microtus) and white mice, while avirulent in guinea pigs and humans, should be attributed to the second subspecies of $Y$. pestis subsp. microtus [6, 7]. This terminology already exists in popular usage [8], and in this review, we will adhere to this variant of intraspecies classification of the causative agent of plague. 
Plague is transmitted to humans predominantly via bites of infected fleas, as well as via direct contact with damaged skin and mucous membranes or via the inhalation of aerosolized respiratory secretions from animals or humans with the pneumonic form of the infection $[2-5]$. In humans, plague occurs as an acute infectious disease manifesting itself as an extremely severe intoxication, fever, lesions in lymph nodes, lungs, and other internal organs, which is frequently complicated with sepsis [4].

The high pathogenicity of $Y$. pestis is to a significant extent due to the unique ability of the bacteria to overcome the defence mechanisms of both mammals and insects, thus ensuring their survival during the entire transmission cycle. Lipopolysaccharide (LPS, endotoxin), the major component of the outer membrane of the cell wall which forms the outer layer of the LPS-phospholipid bilayer, contributes significantly to this feature of the plague microbe. The lipid component of the LPS (the so-called lipid A) acts as an anchor that keeps the LPS molecule bound to the membrane, whereas its carbohydrate chain is oriented towards the environment. A number of pathogenic rough-colony-forming bacteria, including Y. pestis, produce R-type LPS with its carbohydrate moiety being limited to an oligosaccharide (pentasaccharide or higher), which is named the core. The S-type LPS, which is typical of most smooth-colony-forming bacteria, in addition contains a polysaccharide chain (O-antigen) consisting of oligosaccharide repeating units, whereas the core is an intermediate region between the $\mathrm{O}$-antigen and lipid $\mathrm{A}$.

The biosynthesis of the O-antigen and the core-lipid A region proceeds via the independent, but convergent pathways [9]. The initial stages, i.e. the synthesis of lipid A, the transfer of core components to it, and the assembly of the O-antigen repeating unit on an undecaprenol carrier, are performed on the cytoplasmic side of the inner membrane. These stages are followed by the transmembrane transfer. The subsequent stages (polymerization of the repeating unit via the Oantigen-polymerase-dependent pathway, which is the most common pathway in enterobacteria; the possible further modifications in the core-lipid A region and Oantigen; and the linking of both parts into a single molecule) occur on the periplasmic side of the membrane.

LPS plays a significant role in the resistance of bacteria to antibiotics, complement, and other defence systems of the host organism; thus, it can be considered as one of the pathogenicity factors of Gram-negative bacteria. The fine structure of the carbohydrate moiety of LPS determines the specificity of the interaction between a bacterial cell and other biological systems, including the immune system and bacteriophages. Lipid $\mathrm{A}$ is responsible for the majority of the physiologi- cal effects caused by LPS in animals and humans. In mammals, the molecular mechanisms of these effects include the activation of specialized host cells, such as monocytes and macrophages, via the toll-like receptor TLR4, with the participation of the LPS-binding protein and co-receptors CD14 and MD-2. The activated cells secrete nitrogen monoxide, vasoactive lipids and bioactive mediators- pro-inflammatory cytokines. Low concentrations of cytokines are required for triggering the innate immune system of the host; however, their excessive concentration leads to the septic (endotoxic) shock.

Reviews devoted to structural features of the LPS of the plague microbe [10], as well as the immunological properties of its antigens, including LPS [11], have been recently published. This review presents the most up-to-date data pertaining to the chemical composition, structure, genetics and biosynthesis of the LPS of $Y$. pestis, as well as the biological function of the LPS, considered in the context of its structural features. The review also contains a discussion on the possible ways by which the accumulated data pertaining to LPS could be applied in health care.

\section{CHEMICAL COMPOSITION AND STRUCTURE}

The LPS of Y. pestis is composed of a short carbohydrate (oligosaccharide) chain bound to lipid A [10, 12, 13]. This chain contains a conserved pentasaccharide moiety, the so-called inner core, which is typical of all wild strains of enterobacteria. It consists of three residues of L-glycero-D-manno-heptose (LD-Hep) and two residues of 3-deoxy-D-manno-oct-2-ulosonic acid (ketodeoxyoctonic acid, Kdo) (Fig. 1). The inner core of Y. pestis, and certain other enterobacteria (Serratia, Klebsiella, Proteus, Providencia) [14], also contains a Dglucose residue bound to the heptose residue proximal to lipid A (LD-HepI). The aforementioned bacteria form the group with the so-called non-salmonella core type, whereas the salmonella core type contains phosphate, diphosphate, or diphosphoethanolamine, instead of a glucose residue, at the same position of LD-HepI [14]. The inner core region functions as a receptor of most of the bacteriophages specific to the LPS of Y. pestis, including bacteriophage $\varphi \mathrm{A} 1122$ belonging to the T7 group [15, 16], which is used by the U.S. Center for Disease Control and Prevention for phage diagnostics of $Y$. pestis. The glycosidic bond of the Kdo residue located at the reducing terminus of the inner core binds the core to lipid A.

The structure of the $Y$. pestis LPS varies depending upon certain environmental factors; it has been thoroughly studied on preparations isolated from bacteria cultured at different temperatures, serving to imitate the conditions in warm-blooded mammals $\left(37^{\circ} \mathrm{C}\right)$, 
A

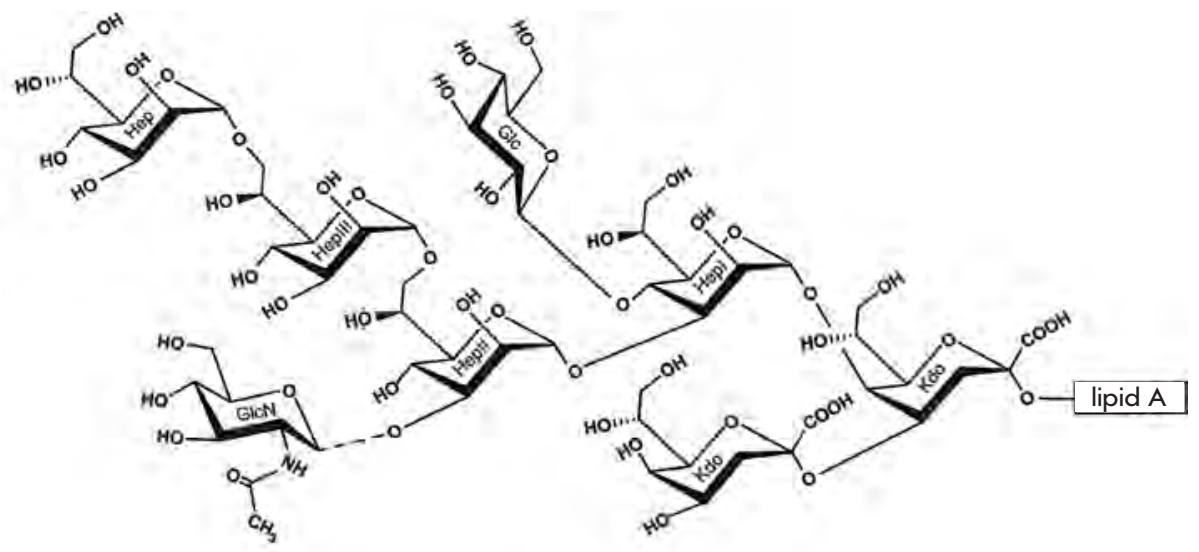

B
Fig. 1. Structural variants of the LPS core of $Y$. pestis $[11-13,17] .(A)$ DD-Hep+Kdo glycoform synthesized as the major variant at $37^{\circ} \mathrm{C}$ and one of the variants produced at $20-28^{\circ} \mathrm{C}$. (B) Gal+Ko glycoform synthesized as one of the variants at $20-28^{\circ} \mathrm{C}$; in addition, DD-Hep+Kdo, DD-Hep+Ko and Gal+Kdo glycoforms are present. (C) DD-Hep+KoPE†N glycoform synthesized at $6^{\circ} \mathrm{C}$; in addition, Gal+Ko PEtN-lacking glycoform is present. Dotted lines indicate nonstoichiometric substitution. Glycine located on LD-Hepl in some strains is not shown.

C
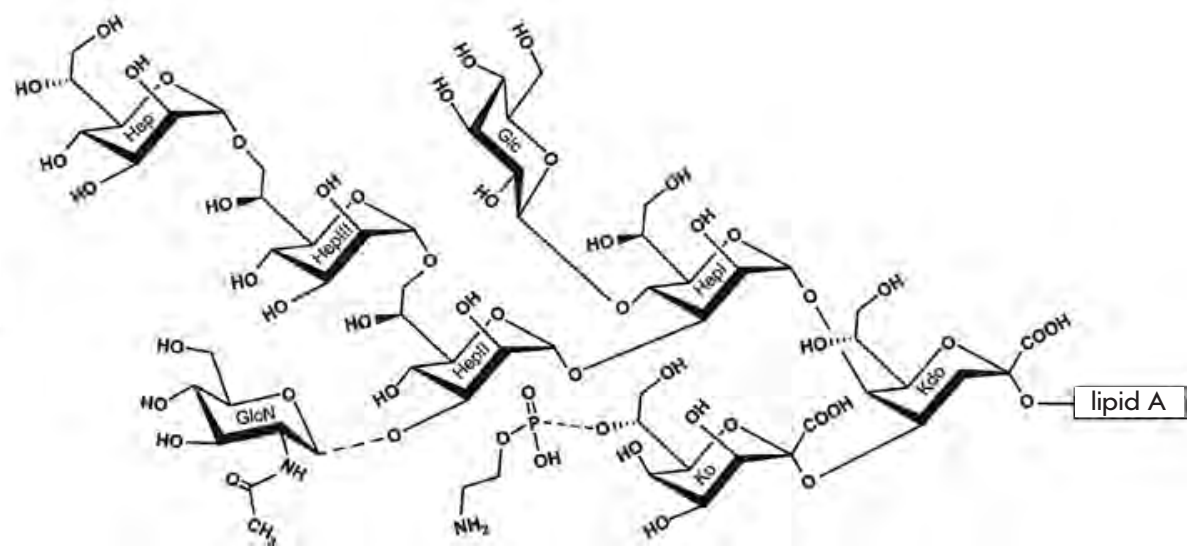

poikilothermic insects $\left(20-28^{\circ} \mathrm{C}\right)$, and hibernating animals $\left(6^{\circ} \mathrm{C}\right)$. The complete inner core is synthesized by both $\mathrm{Y}$. pestis subspecies cultured both at $20-28^{\circ} \mathrm{C}$ and $37^{\circ} \mathrm{C}$. However, the described structure is the sole (or almost sole) glycoform only at $37^{\circ} \mathrm{C}$. (Fig. $\left.1 \mathrm{~A}\right)$, whereas a decrease in temperature results in the partial replacement of the Kdo residue in the side chain by its isosteric 3-hydroxy derivative, the residue of $D$-glycero-
D-malo-oct-2-ulosonic acid (Ko) [12] (Fig. 1B,C). The Ko-containing glycoform is predominant at $6^{\circ} \mathrm{C}$ [17].

$Y$. pestis lacks the outer oligosaccharide region that would be present in the salmonella core type. Its inner region is decorated with several monosaccharides and noncarbohydrate components that are typical of the Yersinia species. Thus, the heptose residue that is most distal from lipid A (LD-HepIII) carries a residue 


\section{REVIEWS}
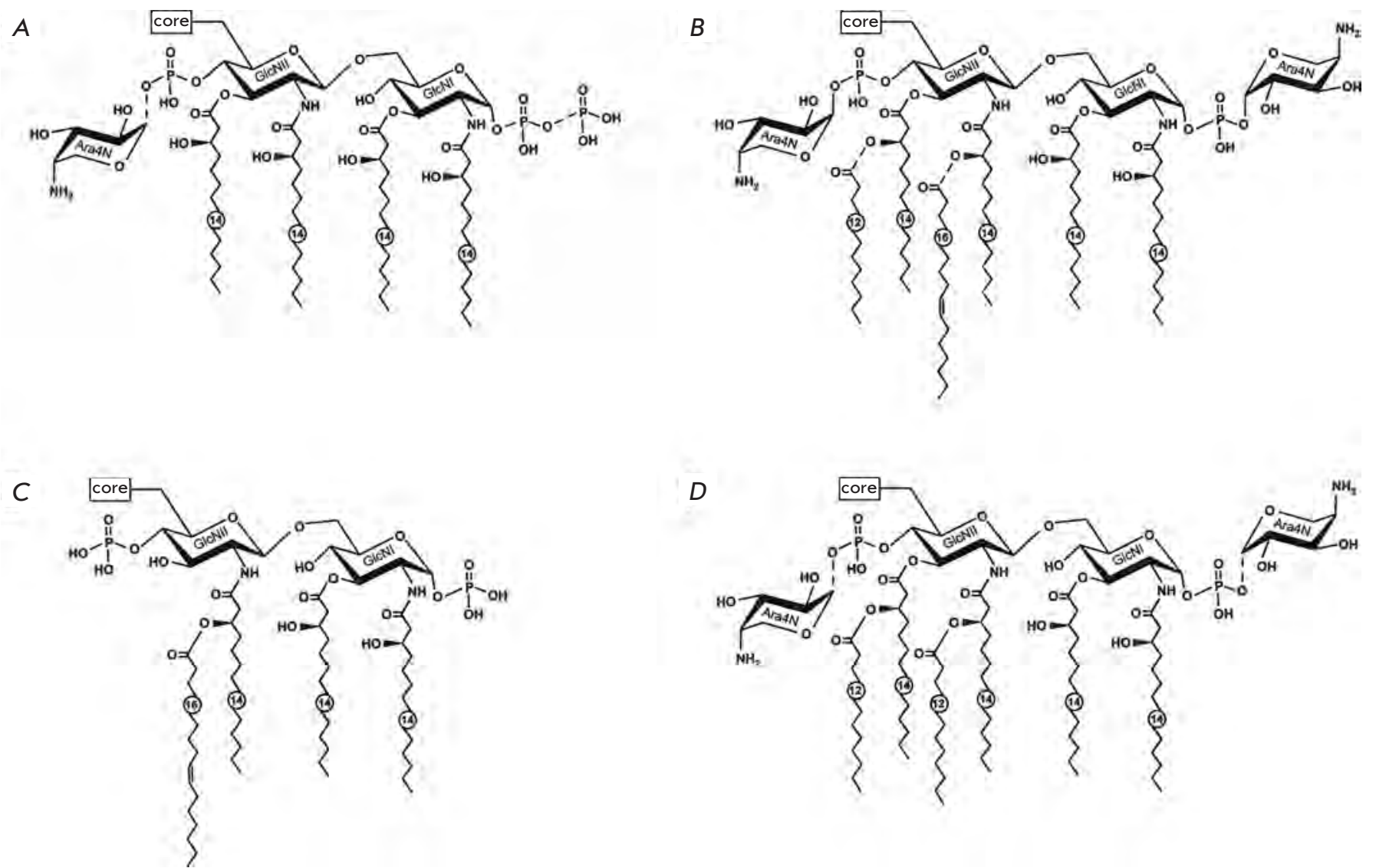

Fig. 2. Structural variants of lipid A of $Y$. pestis. (A) Tetraacyl form synthesized by wild-type strains at $37^{\circ} \mathrm{C}[11,12$, $23,25]$. One of the variants is shown; in the other variants, a diphosphate group may occur at position 4' and Ara4N-1phosphate at position 1; Ara4N-1-diphosphate may replace each of these groups [25]. (B) Hexaacyl form [11, 12, 20, 23] and (C) tetraacyl form [17] synthesized by wild-type strains at $20-28^{\circ} \mathrm{C}$ and $6^{\circ} \mathrm{C}$, respectively. (D) Hexaacyl form synthesized by a recombinant strain of $Y$. pestis bearing the IpxL gene of Escherichia coli at $37^{\circ} \mathrm{C}$ and $26^{\circ} \mathrm{C}[27]$. Dotted lines indicate nonstoichiometric substitution.

of D-glycero-D-manno-heptose (DD-Hep) or D-galactose. The former is typical of the high-temperature LPS variants (Fig. 1A), whereas both variants are synthesized at ambient and decreased temperatures [12] (Figs. 1A,B). Strains of certain biovars (caucasica, altaica) of $Y$. pestis subsp. microtus are incapable of incorporating DD-Hep into the LPS; as a result, most residues of LD-HepIII in the high-temperature LPS forms do not carry any monosaccharide substituents $[12,18,19]$.

The central heptose residue (LD-HepII) is substituted with a residue of $N$-acetyl- $D$-glucosamine, which is present in nonstoichiometric amounts. One of the heptose residues (according to the authors' unpublished data, it is LD-HepI) can be partially acylated with glycine, the content of which decreases with increasing cultivation temperature [12]. The Ko residues are nonstoichiometrically phosphorylated with phosphoethanolamine (PEtN) at $6^{\circ} \mathrm{C}$ [17] (Fig. 1C). $\mathrm{PEtN}$ is also present in certain strains grown at $25^{\circ} \mathrm{C}$ $[18,19]$.

Y. pestis lipid A has a carbohydrate backbone that is typical of enterobacteria and consists of two 1,4'-biphosphorylated glucosamine residues acylated with four residues of 3-hydroxymyristate, which are known as primary acyl groups. Two residues bind to the amino groups, and the two others, to the hydroxyl groups of glucosamine residues (Fig. 2A). Secondary acyl residues, laurate and palmitoleate, bind to the hydroxyl groups of primary fatty acids in the glucosamine residue carrying the core oligosaccharide (GlcNII) $[12,20]$ (Fig. 2B). An additional acyl residue, decanoate, has been detected in $Y$. pestis lipid A; however, its position remains unknown [21, 22].

The content of different acylated forms of lipid A depends to a significant extent on cultivation conditions: it 
is a mixture of tetraacyl, pentaacyl, and hexaacyl forms at $20-28^{\circ} \mathrm{C}$; the triacyl form is also fairly common. A rise in temperature results in a decrease in the degree of acylation of lipid A. Thus, palmitoleate is not bound at $37^{\circ} \mathrm{C}$; therefore, the hexaacyl form is not synthesized at all, and the pentaacyl form with laurate occurs only in a small amount [12, 18, 21, 23].

The high-temperature tetraacyl form (the so-called lipid $\mathrm{IV}_{\mathrm{A}}$ ) contains four primary residues of 3-hydroxymyristate (Fig. 2A), whereas another tetraacyl form with three residues of 3-hydroxymyristate, one of which carries palmitoleate [17] (Fig. 2B), is produced at a decreased temperature $\left(6^{\circ} \mathrm{C}\right)$, along with the hexaacyl form (Fig. 2B). Another feature of Y. pestis lipid A is the cold shock-induced oxidation of one or two of the acyl groups [17]. However, it remains unclear which fatty acids are oxidized and which hydroxylated derivatives are formed during this process.

The phosphate groups of lipid A are glycosylated with residues of a cationic monosaccharide, 4-amino4-deoxy-L-arabinose (Ara4N). In low-temperature LPS variants, the glycosylation of both phosphate groups is almost stoichiometric (Fig. $2 B$ ), whereas the Ara $4 \mathrm{~N}$ content decreases in high-temperature forms $[12,21]$, and one of the phosphate groups is additionally phosphorylated giving rise to a diphosphate group [18, 19, 24, 25] (Fig. 2A). In tetraacyl lipid A of bacteria cultured at $37^{\circ} \mathrm{C}$, the diphosphate group can be located at any of two possible positions. Its presence in the pentaacyl variant at position 4' has been confirmed [25], but its location at position 1 also cannot be excluded. By approximate estimation based on mass spectrometry data, the total diphosphate content in the tetraacyl form is $5-6 \%$. Similarly to monophosphate groups, the diphosphate groups at both positions of lipid A can be partially glycosylated with Ara4N [25]. LPS with the PEtN residue in the core, which is produced at $6^{\circ} \mathrm{C}$, contains no Ara $4 \mathrm{~N}$ in lipid A [17].

The lack of any O-antigen polysaccharide chain is a feature of the Y. pestis LPS that distinguishes it from the LPS of other yersiniae. Meanwhile, similarly to other enterobacteria, Y. pestis can produce the enterobacterial common antigen polysaccharide composed of trisaccharide repeating units comprising one residue of each of the following compounds: $N$-acetyl$D$-glucosamine (GlcNAc), 2-acetamido-2-deoxy-Dmannuronic acid (ManNAcA), and 4-acetamido-4-deoxy-D-fucose (Fuc4NAc), the GlcNAc residue being partially O-acetylated and partially N-deacetylated. From the two known forms of this polysaccharide (the linear form bound to phospholipid or lipid A and the lipid-free cyclic form), in $Y$. pestis the most thoroughly characterized is the cyclic form [26] (Fig. 3A).

\section{A}

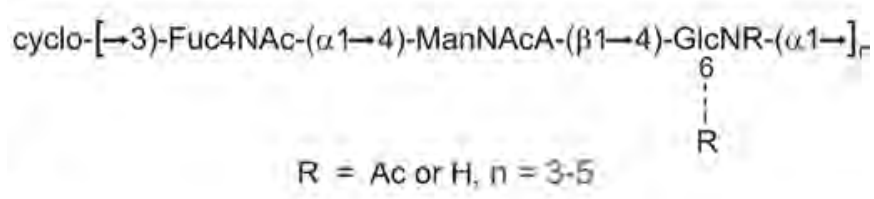

$B$

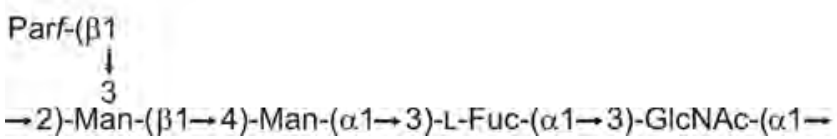

Fig. 3. Structure of the polysaccharide antigens of $Y$. pestis (A) and $Y$. pseudotuberculosis $O: 1 \mathrm{~b}(\mathrm{~B})$. (A) The cyclic form of the common enterobacterial antigen of $Y$. pestis [26]. The glucosamine residue is $\mathrm{N}$-acetylated by $\sim 50 \%$ and 6-O-acetylated by $\sim 20 \%$; $=4$ (major variant), 3 or 5 (minor variants). (B) The pentasaccharide repeating unit of the $O$-antigen of $Y$. pseudotuberculosis $\mathrm{O}: 1 \mathrm{~b}$ [28]. A nonfunctional gene cluster for biosynthesis of this polysaccharide is present in the genome of $Y$. pestis [29]. Par represents 3,6-dideoxy-D-ribo-hexose (paratose). All monosaccharides have the $D$ configuration; paratose occurs in the furanose form, and the other monosaccharides occur in the pyranose form.

\section{GENETICS AND BIOSYNTHESIS}

The tetraacyl biphosphorylated precursor of enterobacterial lipid A (lipid $I_{\mathrm{A}}$ ) in $Y$. pestis is presumed to be synthesized via the same pathway as that of $E$. coli and Salmonella enterica [9], which have been studied most thoroughly in this context. Homologues of the $E$. coli genes that encode the enzymes mediating the late acylation of lipid A (myristoyltransferase LpxM (MsbB), palmitoleyltransferase LpxP and palmitoyltransferase PagP but not lauroyltransferase LpxL (HtrB)), have been identified in the $Y$. pestis genome [22, 30-32].

The functional $l p x M$ and $l p x P$ genes participate in the synthesis of the hexaacyl lipid A of Y. pestis (Fig. 4). Their expression level increases as the cultivation temperature decreases from 37 to $21^{\circ} \mathrm{C}$. Regardless of temperature, the mutant at both genes synthesizes tetraacyl lipid A, which is similar to lipid IV $_{\mathrm{A}}($ Fig. 2A) and is identical to that synthesized by wild-type $Y$. pestis strains at $37^{\circ} \mathrm{C}[22]$. Meanwhile, the transcription level remains low under all conditions. The temperature dependence of the catalytic activity of the enzymes or other post-transcription effects can also affect the lipid A acylation pattern.

$E$. coli acyltransferase LpxIM can use either myristate or laurate as a substrate; however, since its activity is higher with the former substrate, it links myristate to 3-hydroxymyristate at position 3' of GlcNII. This proc- 


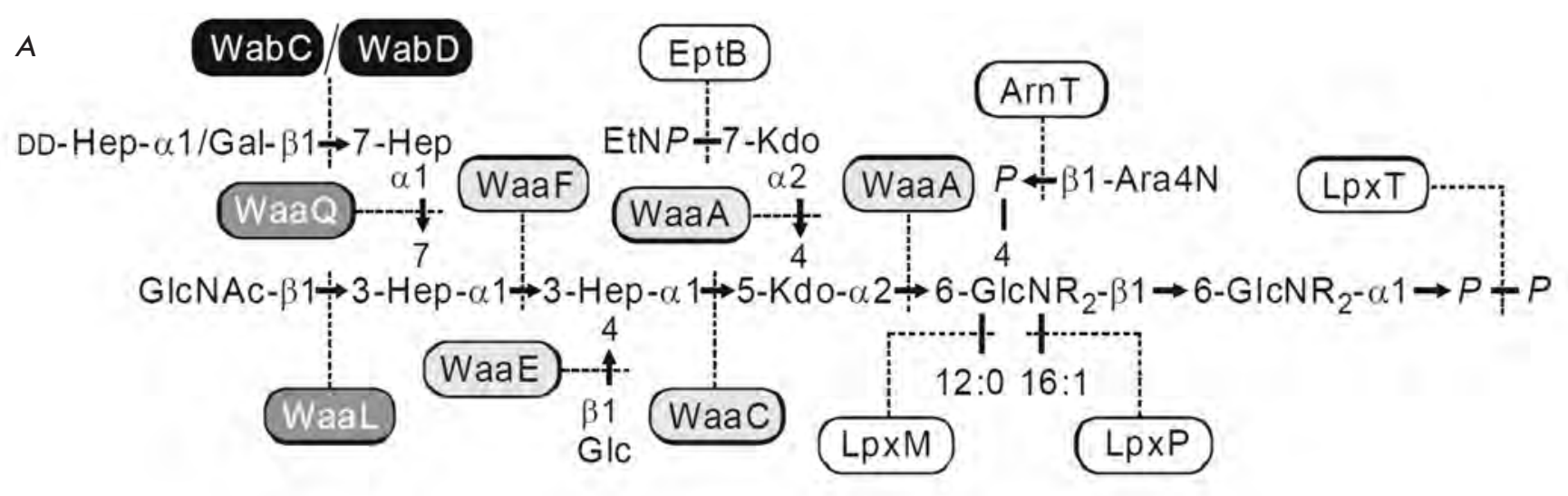

B

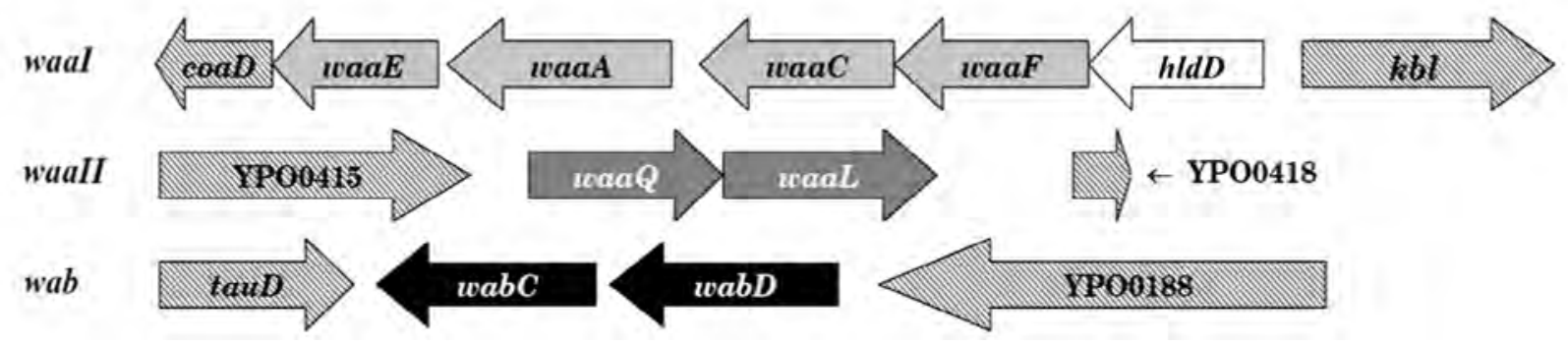

Fig. 4. Biosynthesis of the LPS of $Y$. pestis [22, 32-34]. (A) Enzymes of the LPS core synthesis and the late stages of the lipid A synthesis. R represents 3-hydroxymyristoyl. (B) Organization of the gene clusters for the LPS core synthesis. The genes in the clusters waal (YP0054-YP0058), waall (YP0416-YP0417), and wab (YP0186-YP0187) and the corresponding glycosyl transferases are shown in light gray, dark gray, and black, respectively. The functions of the glycosyl transferases genes are assigned based on the analysis of available $Y$. pestis full genome sequences and the data on LPS structure in knockout mutants at each gene. The figure is reproduced from the paper of the authors [32] with the permission of the Nauka Publishing Company.

ess is preceded by the transfer to 3-hydroxymyristate at position 2' of GlcNII of the secondary acyl group: laurate at $30-42^{\circ} \mathrm{C}$ catalyzed by LpxL [9] or palmitoleate at cold shock temperature $\left(12^{\circ} \mathrm{C}\right)$ with the participation of LpxP [35]. The Y. pestis homologues LpxM and LpxP transfer laurate and palmitoleate to 3-hydroxymyristate residues at positions 3' and 2' GlcNII, respectively. Temperature control was observed only for LpxP, transferring palmitoleate to position 2' GlcNII prior to the binding of the secondary acyl group at position 3' (laurate in Y. pestis or myristate in E. coli), whereas LpxM activity is temperature-independent. Since $Y$. pestis lacks the lpxL gene, 3-hydroxymyristate at position 2' remains unsubstituted at an increased temperature, which deteriorates the efficiency of the laurate transfer with LpxIM and results in the synthesis of mostly the tetraacyl form of lipid A and only a negligible amount of the pentaacyl form. The evidence for this is that the $Y$. pestis recombinant strain KIM5-pLpxL carrying the E. coli lpxL gene produces the hexaacyl form of lipid A with two secondary laurate residues both at 37 and $26^{\circ} \mathrm{C}$ [27] (Fig. 2D).

Acyltransferase PagP in E. coli and S. enterica transfers palmitate from position sn-1 of the glycerophospholipid [36], which distinguishes it from the early and other late acyltransferases that use a substrate bound to the acyl-carrier protein as a donor. Furthermore, palmitoylation of lipid A occurs on the outer rather than the inner membrane [36]. The palmitoylated forms of lipid A are also typical of Y. pseudotuberculosis and $Y$. enterocolitica [19-21] but have not been found in wild-type $Y$. pestis strains, despite the presence of the pag $P$ homologue in the genome, which is $99 \%$ identical to the Y. pseudotuberculosis gene $[31,36]$. The reason for this is the inactivation of this gene due to the substitution of a single nucleotide, resulting in the conversion of a tryptophan-200 codon into a stop codon [31].

The genes encoding the synthesis and transfer of Ara $4 \mathrm{~N}$ are components of the operon arn (pmrHFIJKLM) [9, 37]. An undecaprenyl phosphate (UndP) de- 
rivative synthesized with the participation of Ara4Ntransferase ArnC (PmrF) is a donor of Ara $4 \mathrm{~N}$ for its subsequent binding to the phosphate groups of lipid A. The transfer of Ara4N to lipid A, catalyzed by the product of the arnT ( $p m r K)$ gene, occurs on the periplasmic side of the inner membrane [38]. A complete inner core is required to ensure the most efficient binding of Ara4N, whereas the presence or absence of core monosaccharides distal from lipid A (GlcNAc, Gal and DD-Hep) has virtually no effect on this process [32-34]. Similar to that in E. coli and S. enterica, the arn operon in $Y$. pestis is regulated by two-component signal transduction systems, PhoP/PhoQ and PmrA/PmrB [21, 39]. However, in $Y$. pestis the mechanism of regulation by the PhoP/PhoQ system differs as it occurs without the participation of the PmrD protein, which is absent in this bacteria [39].

The synthesis of the $E$. coli core oligosaccharide begins with the attachment of two Kdo residues to lipid $\mathrm{IV}_{\mathrm{A}}$, a process that is catalyzed by bifunctional Kdo transferase WaaA (Fig. 4A). Kdo transfer precedes the late acylation of lipid A [9]. The subsequent assembly of the core takes place on a completely acylated lipid A and is followed by the transfer of LPS consisting of the core and lipid A through the inner membrane, with the aid of the ABC transporter MsbA. Meanwhile, neither core oligosaccharide nor Kdo (Ko) residues are required for the transmembrane transfer, since LPS without any core (i.e., lipid A) is expressed in the Kdodeficient mutants of E. coli [40,41] and Y. pestis [32, $33,42]$.

The core biosynthesis genes in E. coli, S. enterica, and a number of other enterobacteria are clustered in a chromosomal region, forming the waa cluster [9]. Two clusters (waaI and waaII) with four and two homologues of the waa genes and one cluster with two wab genes, which also encode core biosynthetic enzymes, have been identified in the $Y$. pestis genome [32-34] (Fig. 4B).

The waaI cluster containing most of the genes for the synthesis of the inner core comprises the genes of Kdo transferase WaaA; heptosyltransferases WaaC and WaaF to transfer LD-HepI and LD-HepII, respectively; and glucosyltransferase WaaE. In addition, it includes the gene of heptose 6 -epimerase HldD catalyzing the synthesis of ADP-LD-Hep from its biosynthetic precursor ADP-DD-Hep. Yet another heptosyltransferase gene, waaQ, is located in the waaII cluster. The enzyme encoded by this gene transfers LD-HepIII to LD-HepII; glucose must be present on LD-HepI in order to accomplish the transfer. In turn, the glucose transfer requires the prior attachment of LD-HepII.

The second gene in the cluster waaII is a homologue of the gene of ligase WaaL, which attaches the O-anti- gen to the core [9]. Unlike monosaccharide LPS components transferred by glycosyltransferases in the form of proper nucleoside diphosphate derivatives or (in case of $\mathrm{Kdo}$ ) a nucleoside monophosphate derivative, the undecaprenyl diphosphate (UndPP) derivative acts as a ligase substrate. In the absence of any $\mathrm{O}$-antigen in $\mathrm{Y}$. pestis, WaaL attaches the GlcNAc residue to the core; therefore, this residue is not a true component of the core [12]. Ligase nonstrictly depends on the attachment of Glc and LD-HepIII; without them, the efficiency of the GlcNAc transfer decreases and the LPS of the waaE and waaQ knockout mutants contains only a small amount of this monosaccharide [32, 33]. In Y. pestis and other enterobacteria, the $w e c A$ gene participating in the synthesis of the UndPP-bound GlcNAc is located in the gene cluster of the enterobacterial common antigen [43], whose biosynthesis (in the same manner to that of GlcNAc-containing O-antigens) is initiated by the transfer of GlcNAc-1-phosphate from UDP-GlcNAc to UndP.

The $w a b$ cluster contains the genes of glycosyltransferases WabC and WabD for the transfer of DD-Hep and Gal, respectively. It has been demonstrated using BLAST search that in strains of Y. pestis subsp. microtus bv. caucasica Pestoides F and bv. xilingolensis 91001 , the $w a b C$ gene contains mutations resulting in disturbance of the synthesis of the corresponding protein [32]. Similar mutations are presumably present in the other DD-Hep-defective strains of Y. pestis subsp. microtus bv. caucasica and bv. altaica. The expression of the $w a b D$ gene and/or the activity of the WabD enzyme are temperature-dependent, and the transfer of Gal proceeds inefficiently at increased temperatures. The inability of the phoP mutant to incorporate Gal into the core demonstrates that galactosylation is controlled by the PhoP/PhoQ two-component signal transduction system [44]. Meanwhile, the attachment of DD-Hep does not require the functional PhoP/ PhoQ system.

As mentioned above, the terminal Kdo residue at low temperature is partially substituted for the Ko residue. The latter is synthesized via the oxidation of the 3-deoxy group of $\mathrm{Kdo}$ with a unique $\mathrm{Fe}^{2+} / \alpha$-ketoglutarate/ $\mathrm{O}_{2}$-dependent $\mathrm{Kdo}$-3-hydroxylase ( $\left.\mathrm{KdoO}\right)$ [45]. Its substrate specificity has not been studied; however, taking into account the fact that $\mathrm{KdoO}$ is a peripheral membrane protein, it can reasonably be assumed that 3-hydroxylation of Kdo occurs on the cytoplasmic side of the inner membrane after two Kdo residues have bound to lipid A. The molecular mechanism of the modulation of the temperature-dependent Ko content in the core has as yet to be elucidated.

Gene homologues of transferase EptB (YhjW) transferring PEtN from phosphatidylethanolamine to Kdo 
[32, 33, 46] and phosphatase LpxT (YeiU) transferring phosphate from UndPP to lipid A giving rise to diphosphate $[32,33,47]$ have also been found in the genome of $Y$. pestis $[32,33]$. These genes, in an identical manner to the $k d o O$ gene encoding Kdo-3-hydroxylase, as well as the genes of the late stages of lipid A synthesis (acylation and glycosylation with Ara4N), are spread over the chromosome as individual nonclustered genes. No gene of acyltransferase, which participates in glycine transfer to the LD-HepI residue, has thus far been identified in the genome of $Y$. pestis.

There is 100\% homology of the proteins participating in the LPS biosynthesis (with the exception of the wabC gene that is mutated in a number of representatives of the nonmain subspecies; see above) within the Y. pestis species and 98-100\% homology inside the Yersinia genus [32, 33]. This correlates with the high degree of similarity between the core and lipid A structures of LPS in various Yersinia species [19, 20]. In distantly related bacteria, the homology of the proteins WaaA, WaaC, WaaE, WaaF, EptB, LpxM, LpxP, and ArnT is beyond $70 \%$. Meanwhile, in the enzymes WaaQ, WabC, WaaL, and $\mathrm{KdoO}$, it is lower than $64 \%$, whereas the homology between galactosyltransferase WabD that is unique to the Yersinia species and glycosyltransferases of other bacteria is less than $43 \%$. The high homology of most of the proteins implicated in the LPS biosynthesis in Y. pestis and the bacteria belonging to various phylogenetic groups, in combination with the dispersed location of the corresponding genes in the chromosome of the plague microbe, suggests a multi-stage horizontal transfer of these genes to the genome of the Yersinia progenitor.

A nonfunctional $\mathrm{O}$-antigen gene cluster was identified in the $Y$. pestis genome $[29,48]$. At the nucleotide sequence level, it was $98.9 \%$ identical to the O-antigen cluster in Y. pseudotuberculosis O:1b [29] (the O-antigen structure is shown in Fig. 3B). Therefore, Y. pseudotuberculosis $\mathrm{O}: 1 \mathrm{~b}$ is considered as the most plausible progenitor clone of $Y$. pestis. Of the 17 biosynthetic genes that have been identified in the $\mathrm{O}$-antigen gene cluster of Y. pseudotuberculosis O:1b, five genes in the $Y$. pestis cluster are inactivated by insertions or deletions. These genes include those responsible for the synthesis of the nucleotide-activated derivatives of $L$ fucose and 3,6-dideoxy-D-ribo-hexose (paratose), precursors of the O-antigen components, in the absence of which the O-antigen synthesis is rendered impossible. It is noteworthy that while 16 genes in the clusters of two bacteria are $99-100 \%$ identical, the $w z x$ gene is only $90.4 \%$ identical. This gene encodes flippase Wzx, which mediates the transmembrane transfer of the UndPP-bound pentasaccharide repeating unit of the $\mathrm{O}$-antigen in Y. pseudotuberculosis O:1b. After the loss of this function, Y. pestis flippase presumably changed and centered on the transfer of a single UndPP-bound GlcNAc residue through the inner membrane, which subsequently is bound to the LPS core by ligase WaaL at the same position as the polysaccharide O-antigen in Y. pseudotuberculosis.

\section{BIOLOGICAL PROPERTIES AND ROLE IN PLAGUE PATHOGENESIS}

Production by macrophages and other immune cells of the key pro-inflammatory cytokines (including the tumor necrosis factor $\alpha$ (TNF- $\alpha$ ), the major mediator of septic shock (endotoxemia) that is induced by the action of LPS) plays a significant role in overcoming infectious diseases. In Y. pestis, as in the other Gram-negative bacteria, the cytokine-inducing activity of the LPS mediated by the TLR4 receptor is determined by the lipid A structure [49]. Thus, the production of TNF- $\alpha$ by human and mouse macrophage cell lines considerably falls with a decreasing degree of acylation of lipid A, in particular, with the absence of the hexaacyl form and a significant decrease in the content of the pentaacyl form [18, 23]. These structural changes in lipid A are observed when the temperature of bacterial cultivation increases from $21-28^{\circ} \mathrm{C}$ to $37^{\circ} \mathrm{C}$, which imitates a transition from the temperature conditions in poikilothermic fleas $\left(<30^{\circ} \mathrm{C}\right)$ to those in warm-blooded mammals $\left(37^{\circ} \mathrm{C}\right)[21,23,32$, 33]. In terms of the TNF- $\alpha$ inducing activity at $25^{\circ} \mathrm{C}$, LPS of $l p x M$ knockout mutants occupies an intermediate position between the LPS of the parental strains cultivated at 25 and $37^{\circ} \mathrm{C}$, which is in good agreement with the degree of acylation of lipid A [50].

The limited biological activity of the high-temperature low-acyl form of $Y$. pestis LPS may play a crucial role in overcoming the defence mechanisms of warm-blooded animals by bacteria. Whereas the innate immune system is efficiently stimulated by highacyl LPS forms, the low-acyl forms are not recognized by the TLR4 receptor and, as a result, do no activate the innate immunity via the MD-2-TLR4-dependent pathway. Moreover, in the experiments with human macrophage cell lines [51] and dendritic cells [52], LPS from $Y$. pestis cells cultivated at $37^{\circ} \mathrm{C}$ behaved as an antagonist actively suppressing the TLR4-dependent pro-inflammatory response. The significance of this feature of LPS as a pathogenicity factor of the plague microbe has been convincingly supported by the study of the recombinant $Y$. pestis strain KIM5-pLpxL carrying the E. coli lp $x L$ gene [27]. The LPS of this strain with the 'unnatural' hexaacyl lipid A (Fig. 2D) under all temperature conditions, including $37^{\circ} \mathrm{C}$, stimulates the signalling via TLR 4 and the induction of cytokines (TNF- $\alpha$, interleukines-6 and -8) much more efficiently as compared to LPS of wild-type strains. 
Remarkably, the Y. pestis recombinant strain KIM5pLpxL could not cause bubonic plague in mice, despite the fact that the other pathogenicity factors, such as the type III secretion system, resistance to the bactericidal action of normal serum, and the proteolytic activity of Pla, were not affected. These findings provide a good illustration of the fact that the active (endotoxic) LPS form also plays a positive role in a host, ensuring prompt pathogen recognition and activation of the innate immune system at the early stages of infection. It has been demonstrated on mice model that attenuated $Y$. pestis strains with the immunostimulating LPS form can be regarded as a prototype for a new, efficient live vaccine against plague $[27,53]$.

It should be mentioned that the production of higher acylated low-temperature LPS forms is not a prerequisite for the survival of $Y$. pestis in flea intestine. Thus, the $l p x P / l p x M$ double mutant with tetraacyl lipid A could colonize the digestive tract and block the proventriculus of rat flea Xenopsylla cheopis with the same efficiency as the wild-type strain, which is distinguished by a high level of expression of hexaacyl lipid A in a flea's organism [22].

A decrease in the degree of acylation of lipid A with increasing cultivation temperature [22] or inactivation of the lpxM gene [20] resulted in a moderate or negligible reduction in the lethal toxicity of LPS preparations on the model of mice sensibilized with actinomycin D. The incapacity of the lpxM mutant of $Y$. pestis wildtype strain 231 to synthesize hexaacyl lipid A had no effect on its virulence, whereas the same mutation in the attenuated vaccine strain of $Y$. pestis, EV line NIIEG, reduced its ability to cause lethal infection in mice and guinea pigs $[49,54]$. Importantly, a decrease in the virulence of the $l p x M$ mutant of the vaccine strain was accompanied by a significant increase in its protective activity against bubonic plague as compared with the parental vaccine strain $[49,54]$. This phenomenon can presumably be attributed to the pleiotropic effects of mutation, including changes in the biosynthesis and the exposure character of the major immunoreactive antigens of the bacterial cell surface [55]. If the changes between the mouse and human LPS receptor do not level these changes, inactivation of the $l p x M$ gene can be used for development of a live plague vaccine with reduced adverse effects.

An increase in the cultivation temperature of $Y$. pestis and Y. pseudotuberculosis from 26 to $37^{\circ} \mathrm{C}$ resulted in an increase in the permeability of the outer membrane for the hydrophobic agent $N$-phenyl-1-naphthylamine, which correlated with a decrease in the number (and as a result, with an increase in the flexibility) of the LPS acyl chains [56]. The absence of laurate and palmitoleate made the $l p x P / l p x M$ double mutant sensitive to the deoxycholate detergent, with no effect on its resistance to the hydrophobic antibiotics rifampin and vancomycin [22]. Controversial data have been obtained using cationic antimicrobial peptides (CAMPs), one of the key factors of innate immunity: a decrease in the degree of acylation did not affect the resistance to polymyxin $B$, but it increased the sensitivity to cecropin A [22].

Yet, the resistance to CAMPs depends on the Ara $4 \mathrm{~N}$ content in Y. pestis LPS. This correlation, which is also typical of $S$. enterica and a number of other bacteria [37], can be attributed to the electrostatic repulsion of CAMPs by the cationic monosaccharide, which impedes the binding between the antibiotic molecule and the negatively charged (e.g., phosphate) groups on the outer membrane. A high resistance of the wild-type $Y$. pestis strains with a near-stoichiometric content of Ara $4 \mathrm{~N}$ in the LPS (two Ara $4 \mathrm{~N}$ residues per molecule), which is attained by culturing bacteria at $20-28^{\circ} \mathrm{C}$, has been demonstrated using the polymyxin $\mathrm{B}$ model. A decrease in the resistance to CAMP correlates with a noticeable drop in the content of Ara $4 \mathrm{~N}$ as the temperature is increased to $37^{\circ} \mathrm{C}[21,57]$. Mutants with knockout genes galU encoding the pathway of Ara $4 \mathrm{~N}$ synthesis [58], arnT [32-34] encoding Ara4N-transferase, or phoP $[21,44]$ modulating the binding of Ara $4 \mathrm{~N}$ to lipid A are sensitive to CAMP independently of the cultivation temperature. The role of Ara $4 \mathrm{~N}$ is also supported by a marked increase in the content of this monosaccharide in the LPS of bacteria cultivated at $37^{\circ} \mathrm{C}$ in the presence of polymyxin B [12]. An increase in the Ara $4 \mathrm{~N}$ content in LPS and, as a result, in the resistance of $Y$. pestis to CAMPs with a decreasing cultivation temperature is undoubtedly of adaptive character. High resistance to polymyxin B at a temperature characteristic of insects can presumably be attributed to a greater contribution of CAMPs to the innate immunity defence mechanisms of insects as compared to that of mammals, which have a complement system in addition to CAMPs.

Another cationic component of LPS, glycine [57] located in the core, can contribute to a certain extent to the resistance to CAMPs, whereas uncharged core components do not seem to play a significant role. An increase in the sensitivity to polymyxin $\mathrm{B}$, which was observed for a set of knockout mutants at glycosyltransferase genes producing a truncated core could presumably be attributed to the simultaneous decrease in the Ara $4 \mathrm{~N}$ content in lipid A due to the inefficiency of the Ara $4 \mathrm{~N}$ transfer to LPS molecules with an incomplete carbohydrate moiety [32-34].

The LPS core plays a significant role in the resistance of $Y$. pestis to the complement-mediated bactericidal effect of normal blood serum [32-34], another important component of the innate immune system. Wild-type strains of $Y$. pestis subsp. pestis are resistant 
to normal human serum (NHS) both at $25^{\circ} \mathrm{C}$ and at $37^{\circ} \mathrm{C}$ [57]. The waaL, wabC, wabD, and arnT mutants lacking the terminal core substituents GlcNAc, DD-Hep, Gal or the cationic monosaccharide Ara $4 \mathrm{~N}$, respectively, are characterized by an almost identical resistance. On the contrary, the mutants with an incomplete inner core region are highly susceptible to NHS [32-34]. The molecular mechanism, by which the core contributes to the serum resistance, has not been elucidated thus far. Possibly, it is mediated by the effect of LPS on the folding correctness and, as a result, on the functional activity of the outer membrane protein Ail (OmpX) [59], which plays a crucial role in the resistance of $Y$. pestis to serum [59,60]. Studies of the recombinant E. coli strain carrying the $Y$. pestis ompX gene and its three mutants with a truncated core revealed that the size of the LPS core impacts not only the resistance to NHS, but also the OmpX-mediated virulence factors, such as the adhesive ability and the invasiveness of bacteria [60].

As opposed to strains of the main subspecies, the sensitivity of strains of $Y$. pestis subsp. microtus bv. caucasica to the action of NHS is temperature-independent, which correlates with the absence of documented cases of a human plague caused by strains of this biovar [57]. Meanwhile, these strains are resistant to mouse serum and probably to that of their main host, the common vole, ensuring their survival in rodent blood, which is required for circulation of $Y$. pestis in the natural plague foci. The only distinguishing feature of the LPS of biovar caucasica is that the core contains no DD-Hep [12]. However, a strain of another representative of $Y$. pestis nonmain subspecies, biovar altaica [57], whose LPS also contains no DD-Hep, is as highly resistant to the bactericidal effect of NHS as that of the wabC mutant of the main subspecies with a DD-Hep-deficient LPS [32-34]. This finding suggests that the adaptive changes, which made strains of $Y$. pestis subsp. microtus bv. caucasica sensitive to NHS, affected not only LPS, but also another factor(s) involved within the interaction between a bacterial cell and the complement system.

With no effect on the growth rate of $Y$. pestis cells $[32,33]$, a decrease in the size of the LPS core affects the in vivo formation of a biofilm, the polysaccharidecontaining extracellular matrix, as well as the proventriculus blockage in fleas, which depends on this process [61]. A Y. pestis KIM6+ mutant with the knockout $g m h A$ gene, which encodes one of the enzymes of the LD-Hep biosynthetic pathway, is characterized by a reduced ability to form the biofilm on the cuticle of nematode Caenorhabditis elegans and to block the proventriculus in $X$. cheopis with a moderate decrease in the level of in vitro biofilm formation [61]. This indirect effect of the absence of the major part of the core, including the heptose region, can be attributed to the interaction between the LPS core components and the outer membrane proteins participating in the synthesis, processing, or export of the biofilm.

A significant decrease in the virulence of $Y$. pestis 231 was observed in subcutaneously infected guinea pigs after the LPS core was shortened to five monosaccharide residues and the full loss of virulence for both guinea pigs and mice after the subsequent shortening of the LPS core to three monosaccharides [32, 33]. However, these observations were carried out for a period of 21 days, and it cannot be excluded that a prolongation of the experimental time could have led to the generalization of the infection, resulting in animal death at later stages. Attenuation of the mutants of the virulent CO92 strain of $Y$. pestis with a truncated core was also observed in BALB/c mice, whereas the absence of the core in mutants at the $y r b H$ gene of the Kdo synthesis pathway or the waaA gene encoding Kdo transferase made them completely avirulent [15]. These data prove directly the exceptional significance of the LPS for the pathogenicity of the plague microbe, since an identical dramatic attenuation of $Y$. pestis strains was observed only when the main components of the type III secretion system [4], the gene cluster of synthesis and reception of the siderophore yersiniabactin [4], or the lipoprotein NlpD-encoding genes [62] were lost.

The biological significance of temperature-dependent variations in the monosaccharide composition of the core (replacement of the terminal Kdo and DDHep residues for the Ko and Gal residues, respectively, with decreasing ambient temperature from 37 to $28^{\circ} \mathrm{C}$ and lower) remains to be elucidated. It is reasonable to assume that the hydroxylation of $\mathrm{Kdo}$ at a reduced temperature (i.e., the conversion of Kdo into Ko) compensates for the decrease in the hydrophilicity of the LPS as a result of the acylation of the hydroxyl groups of primary fatty acid residues, which also occurs at low temperatures.

The investigation of the plasminogen activator Pla of $Y$. pestis (an outer membrane protein belonging to the omptin family and exhibiting functions of protease/ adhesin) provided an answer (which may not be the only answer) to the intriguing question of which other advantages (in addition to the trivial energy saving advantage) was acquired by the plague microbe after it had eliminated the necessity for synthesizing the Oantigen. Pla converts plasminogen into plasmin, a key fibrinolytic enzyme, destroys the circulating $\alpha_{2}$-plasmin inhibitor (antiplasmin), induces an uncontrollable tissue proteolysis, and facilitates the dissemination of $Y$. pestis in a macroorganism, thus playing a significant role in a plague pathogenesis. Studies of the activity of $Y$. pestis $\mathrm{Pla}$ in recombinant $\mathrm{Y}$. pseudotuberculosis strains with 
different levels of the O-antigen expression have demonstrated that the O-antigen sterically impedes the interaction between Pla and the high-molecular-mass substrate, thus impeding both plasminogen activation and $\alpha_{2}$-antiplasmin inactivation [63, 64]. This fact leads to the conclusion that the loss of the O-antigen, which is necessary for the enhancement of the enzymatic activity of Pla, has enhanced the invasiveness of Y. pestis. On the other hand, the Pla activity depends on a specific interaction with the phosphate groups of lipid A [65] and requires the presence of LPS with a core that contains at least two LD-Hep residues [32,66]. Such an unusual LPS dependence can be attributed to the fine conformational changes in the active site of omptin due to the binding with LPS [67].

\section{CONCLUSIONS}

A comparison of data pertaining to the structure, biosynthesis, and the biological properties of the LPS in $Y$. pestis and Y. pseudotuberculosis demonstrates that inactivation of the $\mathrm{O}$-antigen gene cluster and the pagP gene, as well as the loss of the $\operatorname{lp} x L$ gene, which results in the synthesis of the R-form of LPS with a short carbohydrate chain and a loss of the ability to produce high-acyl forms of lipid A at $37^{\circ} \mathrm{C}$, were the most significant events during the evolution of $Y$. pestis associated with changes in the LPS structure. These changes play a significant role in the plague pathogenesis, being an important part of the strategy by which the bacteria overcome the host's defence mechanisms. Thus, the absence of the polysaccharide chain ensures the functioning of such a significant pathogenicity factor of $Y$. pestis as the plasminogen activator. The temperature-mediated decrease in the degree of LPS acylation resulting in a reduction of the cytokine-inducing ability is considered to be one of the mechanisms for the prevention of pathogen recognition by the host's immune system at the early stages of infection. These data agree with the conception that the pathoadaptation of $Y$. pestis to a new ecological niche included the loss of the functionality of a number of genes required for saprophytic existence $[36,68]$. The mutations in the $w a b C$ gene encoding DD-Hep-transferase, which remained in representatives of bv. caucasica and bv. xilingolensis of the nonmain subspecies $Y$. pestis subsp. microtus, may have been an element of the subsequent reductive intra-species microevolution during the adaptation of Y. pestis to circulation in the populations of certain vole species.

Meanwhile, a number of LPS features, such as the temperature-dependent variation of both core and lipid A structures, were inherited by Y. pestis from Y. pseudotuberculosis without any noticeable changes. Some of these variations, such as the phosphorylation with
PEtN, the glycosylation with Ara4N, and the oxidation of Kdo into Ko, presumably do not possess any fundamental significance in the new lifestyle of the plague microbe and are required only for the normal functioning of the outer membrane, which is achieved by imparting a certain hydrophilicity and a certain charge to it. On the other hand, they may contribute to plague pathogenesis by facilitating the optimal adaptation of $Y$. pestis at different phases of its existence under the considerably different conditions in mammal hosts and insect vectors. The unique phenomenon of the plague microbe and plague can be a result of the synergic effect of the inherited and newly acquired pathogenicity factors, including those associated with LPS.

Thus, the recent data highlight LPS as a multifunctional pathogenicity factor of $Y$. pestis which plays a key role in the adaptive variability of the plague microbe. However, it should be noted that even if there is a correlation between the LPS structure and the properties of the bacterial culture, the exact biological significance of various LPS modifications cannot be considered completely ascertained, since the cultivation of bacteria in laboratory conditions cannot simulate exactly in vivo conditions. If detailed structures of the LPS synthesized by the bacteria in flea and a warmblooded host were identified, this would enable selection of the in vitro conditions necessary for production of the LPS forms characteristic of a particular infected animal for further laboratory experiments.

The identification of the structure-to-function relationships in Y. pestis LPS opens up new prospects for the design of efficacious live vaccines against plague which are based on the attenuated strains with reduced adverse effects. The approach based on geneticengineering modification of the Y. pestis LPS, which reduces the degree of lipid A acylation, is highly promising. The ability to avoid recognition by the host's defence system at the earliest stage of infection allows the attenuated strain with the mutant LPS to rapidly multiply. However, the absence of one of the major pathogenicity factors as a result of a mutation used for attenuation of a Y. pestis strain prevents the generalization of the infection. This subsequently ensures efficient production of antibodies against the major antigens of the plague microbe and the unset of acquired immunity. Development of efficacious and highly selective next-generation antimicrobial preparations based on high-affinity oligonucleotide ligands can become an alternative approach in plague treatment. Understanding the genetic control of the key stages of the biosynthesis of LPS, intervention in which disturbs the functioning of the outer membrane and attenuates the effect of other virulence factors, will enable to propose new molecular targets for these antibiotics. 
REFERENCES

1. Supotnitskii M.V., Supotnitskaya N.S. Ocherki istorii chumy (Essays of the History of Plague). M.: Vuzovskaya kniga, 2006. Book 1, 468 p.; Book 2. 696 p.

2. Anisimov A.P. // Mol. Gen. Mikrobiol. Virusol. 2002. № 3. P. 3-23.

3. Anisimov A.P. // Mol. Gen. Mikrobiol. Virusol. 2002. № 4. P. 3-11.

4. Perry R.D., Fetherston J.D. // Clin. Microbiol. Rev. 1997. V. 10. P. $35-66$.

5. Anisimov A.P., Lindler L.E., Pier G.B. // Clin. Microbiol. Rev. 2004. V. 17. P. 434-464.

6. Platonov M.E. Molekulyarno-geneticheskoe izuchenie raznoobraziya i mikroevolyutsii Yersinia pestis (Moleculargenetic investigation of diversity and microevolution of Yersinia pestis). PhD thesis. Obolensk, 2010. $142 \mathrm{p}$.

7. Li Y., Cui Y., Hauck Y., Platonov M.E., Dai E., Song Y., Guo Z., Pourcel C., Dentovskaya S.V., Anisimov A.P., et al. // PLoS ONE. 2009. V. 4. P. e6000.

8. Riehm J.M., Vergnaud G., Kiefer D., Damdindorj T., Dashdavaa O., Khurelsukh T., Zöller L., Wölfel R., Le Flèche P., Scholz H.C. // PLoS ONE. 2012. V. 7. P. e30624.

9. Raetz C.R.H., Whitfield C. // Annu. Rev. Biochem. 2002. V. 71. P. $635-700$.

10. Knirel Y.A., Dentovskaya S.V., Senchenkova S.N., Shaikhutdinova R.Z., Kocharova N.A., Anisimov A.P. // J. Endotoxin Res. 2006. V. 12. P. 3-9.

11. Byvalov A.A., Ovodov Yu.S. // Bioorg. Khim. 2011. V. 37. P. 452-463.

12. Knirel Y.A., Lindner B., Vinogradov E.V., Kocharova N.A., Senchenkova S.N., Shaikhutdinova R.Z., Dentovskaya S.V., Fursova N.K., Bakhteeva I.V., Titareva G.M., et al. // Biochemistry. 2005. V. 44. P. 1731-1743.

13. Vinogradov E.V., Lindner B., Kocharova N.A., Senchenkova S.N., Shashkov A.S., Knirel Y.A., Holst O., Gremyakova T.A., Shaikhutdinova R.Z., Anisimov A.P. // Carbohydr. Res. 2002. V. 337. P. 775-777.

14. Holst O. Endotoxin in Health and Disease. N.Y.: Marcel Dekker, 1999. P. 115-154.

15. Filippov A.A., Sergueev K.V., He Y., Huang X.Z., Gnade B.T., Mueller A.J., Fernandez-Prada C.M., Nikolich M.P. // PLoS ONE. 2011. V. 6. P. e25486.

16. Kiljunen S., Datta N., Dentovskaya S.V., Anisimov A.P., Knirel Y.A., Bengoechea J.A., Holst O., Skurnik M. // J. Bacteriol. 2011. V. 193. P. 4963-4972.

17. Knirel Y.A., Lindner B., Vinogradov E.V., Shaikhutdinova R.Z., Senchenkova S.N., Kocharova N.A., Holst O., Pier G.B., Anisimov A.P. // Carbohydr. Res. 2005. V. 340. P. 1625-1630.

18. Dentovskaya S.V., Bakhteeva I.V., Titareva G.M., Shaikhutdinova R.Z., Kondakova A.N., Bystrova O.V., Lindner B., Knirel Y.A., Anisimov A.P. // Biochemistry (Moscow). 2008. V. 73. P. 192-199.

19. Knirel Y.A., Kondakova A.N., Bystrova O.V., Lindner B., Shaikhutdinova R.Z., Dentovskaya S.V., Anisimov A.P. // Adv. Sci. Lett. 2008. V. 1. P. 192-198.

20. Aussel L., Thérisod H., Karibian D., Perry M.B., Bruneteau M., Caroff M. // FEBS Lett. 2000. V. 465. P. 87-92.

21. Rebeil R., Ernst R.K., Gowen B.B., Miller S.I., Hinnebusch B.J. // Mol. Microbiol. 2004. V. 52. P. 1363-1373.

22. Rebeil R., Ernst R.K., Jarrett C.O., Adams K.N., Miller S.I., Hinnebusch B.J. // J. Bacteriol. 2006. V. 188. P. 1381-1388.

23. Kawahara K., Tsukano H., Watanabe H., Lindner B., Matsuura M. // Infect. Immun. 2002. V. 70. P. 4092-4098.
24. Jones J.W., Shaffer S.A., Ernst R.K., Goodlett D.R., Tureček F. // Proc. Natl. Acad. Sci. USA. 2008. V. 105. P. 12742-12747.

25. Jones J.W., Cohen I.E., Tureček F., Goodlett D.R., Ernst

R.K. // J. Am. Soc. Mass Spectrom. 2010. V. 21. P. 785-799.

26. Vinogradov E.V., Knirel Y.A., Thomas-Oates J.E.,

Shashkov A.S., L'vov V.L. // Carbohydr. Res. 1994. V. 258. P. 223-232.

27. Montminy S.W., Khan N., McGrath S., Walkowicz M.J., Sharp F., Conlon J.E., Fukase K., Kususmoto S., Sweet C., Miyake K., et al. // Nat. Immunol. 2006. V. 7. P. 1066-1073.

28. Kondakova A.N., Shaikhutdinova R.Z., Ivanov S.A., Dentovskaya S.V., Shashkov A.S., Anisimov A.P., Knirel Y.A. // Carbohydr. Res. 2009. V. 344. P. 2421-2423.

29. Skurnik M., Peippo A., Ervelä E. // Mol. Microbiol. 2000. V. 37. P. $316-330$.

30. Dentovskaya S.V., Shaikhutdinova R.Z., Knirel Y.A., Ivanov S.L., Anisimov A.P. // Mol. Gen. Mikrobiol. Virusol. 2006. № 2. P. 3-8.

31. Bishop R.E. // Mol. Microbiol. 2005. V. 57. P. 900-912.

32. Dentovskaya S.V., Anisimov A.P., Kondakova A.N., Bystrova O.V., Lindner B., Svetoch T.E., Shaikhutdinova R.Z., Ivanov S.A., Bakhteeva I.V., Titareva G.M., Knirel Y.A. // Biochemistry (Moscow). 2011. V. 76. P. 808-822. 33. Anisimov A.P., Dentovskaya S.V., Kondakova A.N., Lindner B., Shaikhutdinova R.Z., Kocharova N.A., Senchenkova S.N., Knirel Y.A. The Challenge of Highly Pathogenic Microorganisms - Mechanism of Virulence and Novel Medical Countermeasures. Dordrecht: Springer, 2010. P. 77-87.

34. Knirel Y.A., Dentovskaya S.V., Bystrova O.V., Kocharova N.A., Senchenkova S.N., Shaikhutdinova R.Z., Titareva G.M., Bakhteeva I.V., Lindner B., Pier G.B., Anisimov A.P. // Adv. Exp. Med. Biol. 2007. V. 603. P. 88-96.

35. Carty S.M., Sreekumar K.R., Raetz C.R.H. // J. Biol. Chem. 1999. V. 274. P. 9677-9685.

36. Bishop R.E., Gibbons H.S., Guina T., Trent M.S., Miller S.I., Raetz C.R. // EMBO J. 2000. V. 19. P. 5071-5080.

37. Gunn J.S., Lim K.B., Krueger J., Kim K., Guo L., Hackett M., Miller S.I. // Mol. Microbiol. 1998. V. 27. P. 1171-1182. 38. Trent M.S., Ribeiro A.A., Doerrler W.T., Lin S., Cotter R.J., Raetz C.R.H. // J. Biol. Chem. 2001. V. 276. P. 4313243144 .

39. Winfield M.D., Latifi T., Groisman E.A. // J. Biol. Chem. 2005. V. 280. P. 14765-14772.

40. Meredith T.C., Aggarwal P., Mamat U., Lindner B.,

Woodard R.W. // ACS Chem. Biol. 2006. V. 1. P. 33-42.

41. Reynolds C.M., Raetz C.R. // Biochemistry. 2009. V. 48. P. 9627-9640.

42. Tan L., Darby M. // J. Bacteriol. 2005. V. 187. P. 6599-6600. 43. Pacinelli E., Wang L., Reeves P.R. // Infect. Immun. 2002. V. 70. P. 3271-3276.

44. Hitchen P.G., Prior J.L., Oyston P.C., Panico M., Wren B.W., Titball R.W., Morris H.R., Dell A. // Mol. Microbiol. 2002. V. 44. P. 1637-1650.

45. Chung H.S., Raetz C.R. // Proc. Natl. Acad. Sci. USA. 2011. V. 108. P. $510-515$.

46. Reynolds C.M., Kalb S.R., Cotter R.J., Raetz C.R. // J. Biol. Chem. 2005. V. 280. P. 21202-21211.

47. Touze T., Tran A.X., Hankins J.V., Mengin-Lecreulx D., Trent M.S. // Mol. Microbiol. 2008. V. 67. P. 264-277.

48. Prior J.L., Parkhill J., Hitchen P.G., Mungall K.L., Stevens K., Morris H.R., Reason A.J., Oyston P.C.F., Dell A., Wren B.W., Titball R.W. // FEMS Microbiol. Rev. 2001. V. 197. P. 229-233. 


\section{REVIEWS}

49. Park B.S., Song D.H., Kim H.M., Choi B.S., Lee H., Lee J.O. // Nature. 2009. V. 458. P. 1191-1195.

50. Anisimov A.P., Shaikhutdinova R.Z., Pan'kina L.N., Feodorova V.A., Savostina E.P., Bystrova O.V., Lindner B., Mokrievich A.N., Bakhteeva I.V., Titareva G.M., et al. // J. Med. Microbiol. 2007. V. 56. P. 443-453.

51. Matsuura M., Takahashi H., Watanabe H., Saito S., Kawahara K. // Clin. Vaccine Immunol. 2010. V. 17. P. 49-55.

52. Telepnev M.V., Klimpel G.R., Haithcoat J., Knirel Y.A., Anisimov A.P., Motin V.L. // J. Infect. Dis. 2009. V. 200. P. 1694-1702.

53. Sun W., Six D., Kuang X., Roland K.L., Raetz C.R.H., Curtis R. III. // Vaccцne. 2011. V. 29. P. 2986-2998.

54. Feodorova V.A., Pan'kina L.N., Savostina E.P., Sayapina L.V., Motin V.L., Dentovskaya S.V., Shaikhutdinova R.Z., Ivanov S.A., Lindner B., Kondakova A.N., et al. // Vaccine. 2007. V. 25. P. 7620-7628.

55. Feodorova V.A., Pan'kina L.N., Savostina E.P., Kuznetsov O.V., Konnov N.P., Sayapina L.V., Dentovskaya S.V., Shaikhutdinova R.Z., Ageev S.A., Lindner B., et al. // Vaccine. 2009. V. 27. P. 2240-2250.

56. Bengoechea J.A., Brandenburg K., Seydel U., Díaz R., Moriyón I. // Microbiology. 1998. V. 144. P. 1517-1526.

57. Anisimov A.P., Dentovskaya S.V., Titareva G.M., Bakhteeva I.V., Shaikhutdinova R.Z., Balakhonov S.V., Lindner B., Kocharova N.A., Senchenkova S.N., Holst O., et al. // Infect. Immun. 2005. V. 73. P. 7324-7331.

58. Klein K.A., Fukuto H.S., Pelletier M., Romanov G., Grabenstein J.P., Palmer L.E., Ernst R., Bliska J.B. // J. Bacteriol. 2012. V. 194. P. 653-662.
59. Bartra S.S., Styer K.L., O’Bryant D.M., Nilles M.L., Hinnebusch B.J., Aballay A., Plano G.V. // Infect. Immun. 2008. V. 76. P. $612-622$.

60. Kolodziejek A.M., Schnider D.R., Rohde H.N., Wojtowicz A.J., Bohach G.A., Minnich S.A., Hovde C.J. // Infect. Immun. 2010. V. 78. P. 5233-5243.

61. Darby C., Ananth S.L., Tan L., Hinnebusch B.J. // Infect. Immun. 2005. V. 73. P. 7236-7242.

62. Tidhar A., Flashner Y., Cohen S., Levi Y., Zauberman A., Gur D., Aftalion M., Elhanany E., Zvi A., Shafferman A., et al. // PLoS ONE. 2009. V. 4. P. e7023.

63. Kukkonen M., Suomalainen M., Kyllonen P., Lahteenmaki K., Lang H., Virkola R., Helander I.M., Holst O., Korhonen T.K. // Mol. Microbiol. 2004. V. 51. P. 215-225.

64. Pouillot F., Derbise A., Kukkonen M., Foulon J., Korhonen T.K., Carniel E. // Microbiology. 2005. V. 151. P. 3759-3768.

65. Suomalainen M., Lobo L.A., Brandenburg K., Lindner B., Virkola R., Knirel Y.A., Anisimov A.P., Holst O., Korhonen T.K. // Infect. Immun. 2010. V. 78. P. 2644-2652.

66. Dentovskaya S.V., Platonov M.E., Bakhteeva I.V., Anisimov A.P. Problemy osobo opasnykh infektsii (Probl. Particularly Dangerous Infect. (Saratov)) 2007. V. 93. P. 49-51. 67. Eren E., Murphy M., Goguen J., van den Berg B. // Structure. 2010. V. 18. P. 809-818.

68. Chain P.S., Carniel E., Larimer F.W., Lamerdin J., Stoutland P.O., Regala W.M., Georgescu A.M., Vergez L.M., Land M.L., Motin V.L., et al. // Proc. Natl. Acad. Sci. USA. 2004. V. 101. P. 13826-13831. 